\title{
Desenvolvimento de um software PIMS com comunicação OPC e acesso por navegador Web
}

\author{
Silas M. Sousa* Michelle M. Santos** \\ Isabel R. H. Oliveira ${ }^{* * *}$ \\ * Centro Federal de Educação Tecnológica de Minas Gerais, Belo \\ Horizonte, MG; Instituto Federal de Minas Gerais, Ouro Preto, MG, \\ (e-mail: silas.sousa@ifmg.edu.br) \\ ** Instituto Federal de Minas Gerais, Betim, MG (e-mail: \\ michelle.mendes@ifmg.edu.br) \\ *** Universidade Federal de São João del Rei, São João del-Rei, MG, \\ (e-mail: isabelrho@hotmail.br)
}

\begin{abstract}
Automation systems have a range of information inherent in the industrial process. This information can help the corporate sectors of the industry in making strategic decisions. This paper presents the development of a tool for obtaining and storing data from automation systems, known as PIMS. The software developed in this work uses the OPC Standard allowing communication with the main manufacturers of PLC. This information is stored in a SQL Server database. Through a Web site, also developed in the work, various sectors of the industry can visualize the data in graphs and tables, generate reports of data and export them in PDF, DOC and XLS formats. The software proposes a cost-effective solution to small and medium size industries in managing process information. The tests showed that the software was able to get the information and reproduce the values in a Web interface.

Resumo: Os sistemas de automação detêm uma gama de informações inerentes ao processo industrial. Essas informações podem ajudar os setores corporativos da indústria na tomada de decisões estratégicas. O presente trabalho trata do desenvolvimento de uma ferramenta de obtenção e armazenamento de dados dos sistemas de automação, conhecido como PIMS. O software desenvolvido neste trabalho utiliza o Padrão OPC permitindo a comunicação com os principais fabricantes de CLP. Essas informações são armazenadas em um banco de dados SQL Server. Por meio de um Web Site, também desenvolvido no trabalho, diversos setores da indústria podem visualizar os dados em gráficos e tabelas, gerar relatórios de dados e exportá-los nos formatos PDF, DOC e XLS. O software propõe uma solução economicamente viável para empresas de pequeno e médio porte no gerenciamento das informações do processo. Os testes mostraram que o software foi capaz de obter as informações e reproduzir os valores em uma interface $W e b$.
\end{abstract}

Keywords: CLP; Database; OPC standard; PIMS software; SQL Server.

Palavras-chaves: Banco de dados; CLP; Padrão OPC; PIMS; SQL Server.

\section{INTRODUÇÃO}

A informação é uma ferramenta importante para gerenciar os ativos de uma planta industrial como a qualidade do produto, quantidade de matéria prima e parâmetros do processo de produção. Segundo Stair and Reynolds (2002) "O valor da informação está diretamente ligado à maneira como ela ajuda os tomadores de decisões a atingirem as metas da organização".

Com o advento da indústria inteligente, chamada "Indústria 4.0", a informação assume papel principal integrando todos os setores de uma indústria a fim de alcançar o aumento da produção, redução de custos, eficiência energética e visão nas mudanças tecnológicas. Para isso a plata- forma técnica deve estar estruturada com redes industriais, sistemas de otimização e banco de dados (Jazdi, 2016).

Por meio de informações os setores corporativos de uma indústria podem embasar suas decisões em dados reais do processo, que estão disponíveis em sua tela de computador ou até mesmo dispositivos mobile (Sousa and Santos, 2014).

Cada setor da empresa tem um interesse específico em determinada informação. A manutenção precisa monitorar a vida útil dos motores com base nos dados de vibração e temperatura. Para o setor de produção, parâmetros como a qualidade e o consumo de matéria prima são essenciais para seu trabalho. Os dados de processo podem ser utilizados para medir índices de produção como é o caso do Overall Equipment Effectiveness (OEE) que 
mede a eficiência de equipamentos levando em conta três critérios: disponibilidade, desempenho e qualidade (Kao et al., 2016).

É imprescindível que os setores corporativos se comuniquem com o chão de fábrica, para isso são utilizadas as redes industriais que permitem a troca de informação. A Manufatura Inteligente é a utilização dos dados de produção em benefício dos setores corporativos de uma empresa (Dang, 2007).

A pirâmide da automação, Figura 1, mostra três níveis diferentes de informações. O primeiro é o nível de controle e supervisão, onde estão presentes os equipamentos que interagem diretamente com o processo, como instrumentação, CLP (Controlador Lógico Programável) e SCADA (Supervisory Control and Data Acquisition). O segundo nível é representado pelas ferramentas de gerenciamento da produção, PIMS (Process Information Management System) e MES (Manufacturing Execution System). PIMS é um sistema para armazenamento de informações do processo com ferramentas para análise de desempenho (Carvalho et al., 2003). O MES é um sistema que auxilia no planejamento da produção. O último nível é representado pelo ERP (Enterprise Resource Planning), que engloba os diversos setores da empresa como estoque, financeiro, logística, produção e administração, auxiliando no gerenciamento de negócios.

A Figura 2 apresenta o fluxo de informações através dos níveis da pirâmide de automação. Quanto mais elevado é o nível na pirâmide mais filtrados e informativos ficam os dados, consequentemente o volume de dados diminui. Desta forma os dados brutos são transformados em informação útil, gerando conhecimento.

Muitas indústrias de pequeno e médio porte ainda possuem sua solução de automação voltada apenas a CLP e SCADA. Porém, cada vez mais, essas indústrias têm se mostrado a favor da manufatura inteligente para conseguirem melhores resultados. Os softwares existentes no mercado que desempenham essa função, como por exemplo, o

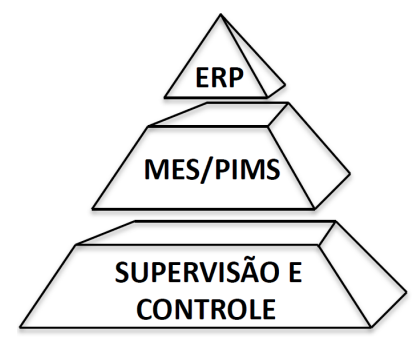

Figura 1. Pirâmide da automação com três níveis de informações do processo. Adaptado de (Seixas, 2005).

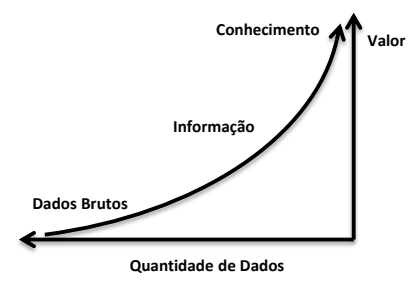

Figura 2. Concentração dos dados em uma empresa e quantidade de dados. Adaptado de (Seixas, 2005).
InfoPlus e PI System, não são acessíveis a essas indústrias. Surge, então, uma lacuna no mercado com a necessidade de um software PIMS de baixo custo, que oferte o suporte necessário para obter, armazenar e mostrar os dados do processo.

Até a década de 1990, cada fabricante de dispositivos para automação possuíam drives proprietários para realizar a comunicação entre seus dispositivos. Isso dificultava a utilização de dispositivos de diferentes fabricantes em um mesmo sistema. Para resolver esse problema empresas deste seguimento se uniram e criaram um protocolo chamado de OPC (OLE for Process Control). Com um protocolo padrão reduziram-se custos e tempo, além de promover a interoperabilidade (Shimanuki, 1999). A comunicação OPC é o padrão utilizado por mais de 300 fabricantes de equipamentos para automação e sistemas de controle (Tan et al., 2007).

A Tabela 1 mostra os valores de venda de alguns PIMS utilizados em sistemas de automação. Todos os PIMS mostrados tem a função de comunicação OPC e geram relatórios de dados.

Tabela 1. Preços e funções dos historiadores PIMS do mercado. Fonte: (Control, 2014) e

(Tierney, 2013).

\begin{tabular}{cc} 
Produto (Empresa) & Preço \\
\hline Infoplus 21 (Aspentech) & $\mathrm{R} \$ 120.000 /$ ano \\
PI System (OSI Software) & $\mathrm{R} \$ 600.000\left(1^{\circ}\right.$ ano) + \\
& $\mathrm{R} \$ 120.000 /$ ano \\
Wondeware Historian (Wonderware) & $\mathrm{R} \$ 20.995$ (só licença) \\
\hline
\end{tabular}

Os valores referentes ao Infoplus 21 (da empresa Aspentech) e PI System (da OSI Software) contemplam a licença e a instalação/manutenção. A empresa Wonderware não presta os serviços de instalação/manutenção do PIMS, portanto o valor de venda do Wonderware Historian se refere apenas à licença comercial.

O objetivo deste trabalho é mostrar o desenvolvimento de um sistema de aquisição dos dados do processo industrial e seu armazenamento em banco de dados, disponibilizandoos aos gestores por meio de gráficos, tabelas e relatórios. Para testar o software criado neste trabalho foi utilizada uma planta didática de controle de temperatura e nível de tanques.

\section{DESENVOLVIMENTO DO SOFTWARE PIMS}

O sistema desenvolvido é composto de duas partes. A primeira é um software executável cuja função é realizar a comunicação com os sistemas de automação por meio do padrão OPC e obter os dados do processo (Cliente OPC). Esse software também deve armazenar os dados em um banco de dados. A segunda parte do sistema é um Web Site utilizado para visualização dos gráficos e tabelas.

\subsection{Executável local: Servidor do software PIMS}

A primeira etapa de desenvolvimento do sistema consiste em criar um Cliente OPC, assim será estabelecido a comunicação com um Servidor OPC para buscar os dados do processo. Para criar aplicações com comunicação OPC é necessário ter profundo conhecimento das regras 
do padrão, contidas nas cartilhas da OPC Foundation (OPC Foundation, 2018). Para facilitar o uso do OPC, desenvolvedores traduziram esse conjunto de normas e especificações em bibliotecas. Essas bibliotecas são disponibilizadas no formato Dynamic Link Libraries (DLL) e assim podem ser utilizadas mais facilmente, diminuindo o tempo de desenvolvimento da aplicação. As bibliotecas utilizadas para interpretar o padrão OPC são:

- OpcNetApi.dll;

- OpcNetApi.Com.dll;

- OpcNetApi.Xml.dll.

Essas bibliotecas foram disponibilizadas no site da Mesta Automation (Mesta, 2014). Elas foram desenvolvidas para a linguagem $\mathrm{C} \#$ e para seu correto funcionamento devem ser declaradas no preâmbulo do código: using Opc.Da.

O Padrão OPC classifica seus objetos em Item (que contém a informação de uma variável de processo), Grupo (conjunto de itens) e o Servidor (fonte de dados). Durante a conexão o Cliente OPC cria um grupo de leitura de itens. Cada item deve ser adicionado ao grupo OPC para que sua leitura seja feita periodicamente. Também é necessário indicar o tipo de dado como, por exemplo, inteiro, binário, decimal ou texto. A cada período de atualização do Cliente OPC a função de tratamento dos dados é chamada. Essa função é responsável por dar algum destino aos dados obtidos. No caso do executável local, a cada atualização os dados são armazenados no banco de dados SQL (Microsoft, 2019).

Para realizar varreduras periódicas dos valores dos itens OPC é utilizado um temporizador (timer). Ele especifica a taxa de atualização dos dados. Cada aplicação pode ter uma necessidade diferente quanto ao tempo de atualização. Caso a taxa seja muito alta o desempenho do sistema pode ficar comprometido, bem como a capacidade do armazenador de dados.

Ao adicionar um item OPC o executável local cria uma tabela no banco de dados para armazenar as informações dessa variável, contendo as colunas data time e valor (conforme Tabela 2). O banco de dados utilizado foi o Microsoft SQL Server.

Tabela 2. Tabela do banco de dados para armazenar informações do item nível $[\mathrm{m}]$.

\begin{tabular}{cc} 
data_time & valor \\
\hline $03 / 01 / 2018$ 09:15:09 & 78,90 \\
$03 / 01 / 2018$ 09:15:10 & 78,92 \\
03/01/2018 09:15:11 & 79,04 \\
$03 / 01 / 201609: 15: 12$ & 78,98 \\
\hline
\end{tabular}

Para realizar as ações envolvendo o banco de dados são utilizados comandos de consulta (querys). Para conectar ao banco de dados é utilizado o comando SQL: SqlConnection con = new SqlConnection ("Data Source=ServidorSQL; User $I D=$ Login; Password=Senha"), em que con é a variável de conexão e o texto entre parênteses é a string de conexão. É nessa string que são passados os parâmetros de Nome do Servidor SQL, Login e Senha do Usuário.

O executável local foi desenvolvido no programa Visual Studio e possui a programação do Cliente OPC e Banco de Dados. As telas de navegação são utilizadas para configurar o servidor do software PIMS.

A Figura 3 mostra a tela de configuração do banco de dados, onde é possível testar a conectividade com o banco. Na parte inferior da Figura 3 pode-se observar a opção "Criar banco de dados e Tabelas". Esse comando cria todas configurações necessárias para o correto funcionamento do software PIMS, evitando que o usuário configure de forma incorreta algum parâmetro do banco de dados.

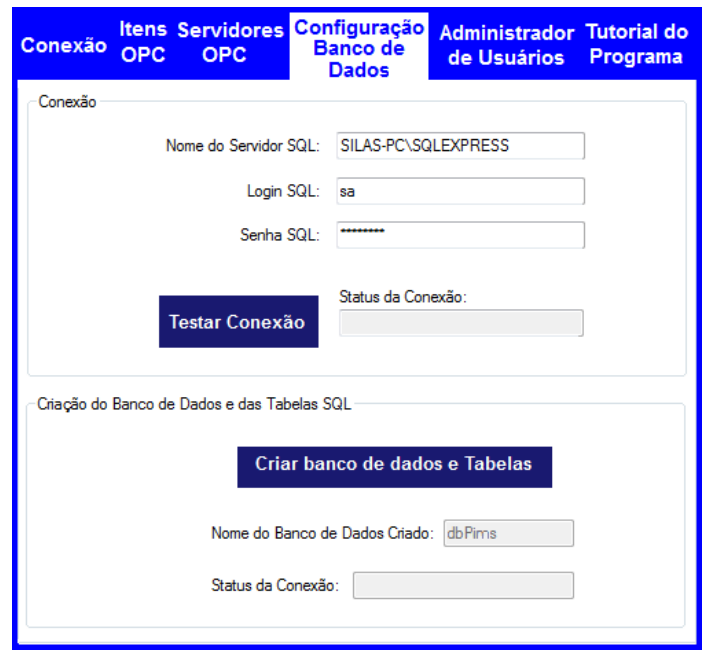

Figura 3. Tela de Configuração de Conexão ao Banco de Dados.

Na Figura 3 também é possível observar o menu de acesso às outras telas do executável local:

- Conexão com o Servidor OPC;

- Itens OPC;

- Servidor OPC;

- Controle de acesso e logins;

- Tutorial de configuração do Servidor do software PIMS.

Por meio das telas, o usuário pode realizar as configurações do executável local conforme seu processo industrial. A tela "Tutorial do Programa" funciona como uma ajuda na configuração do software PIMS, para usuários que ainda não estão familiarizados com o executável local.

\subsection{Web Site de visualização dos dados}

O Web Site do software PIMS acessa a base de dados SQL e apresenta os dados do processo ao usuário por meio de gráficos e tabelas. Para isso o usuário irá escolher as variáveis e também o período de visualização da informação.

O Web Site também foi feito em Visual Studio e conta com três ambientes de programação: Código, Visual e HTML. No ambiente de código é configurado o acesso ao banco de dados. O ambiente Visual é utilizado para desenvolver sua estrutura visual (layout). Para configurar as funcionalidades do Web Site, como a plotagem de gráficos e tabelas, é utilizado o ambiente de HTML. A Figura 4 mostra a tela de visualização dos gráficos.

Por meio da interface gráfica é possível exibir gráficos de linha, coluna e de pizza. Outra informação importante que 

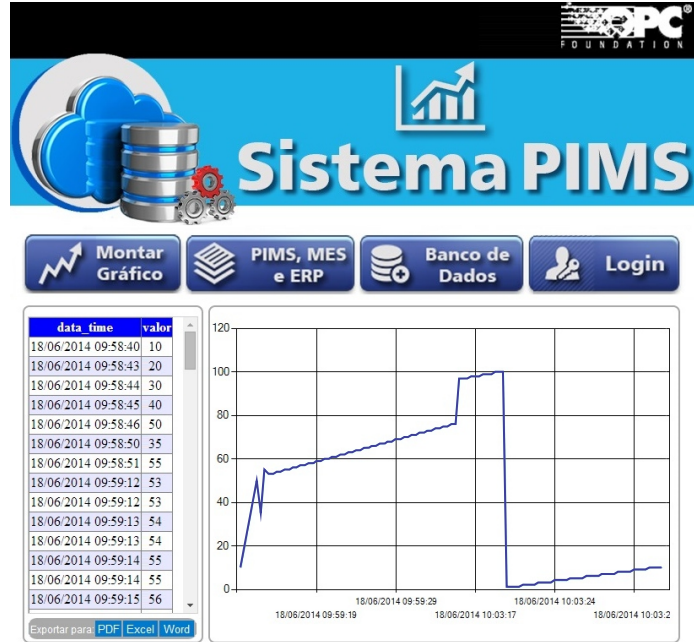

Figura 4. Tela de visualização de gráficos do Web Site do software PIMS.

pode ser visualizada é a porcentagem de tempo de parada de uma máquina ou equipamento.

\section{SISTEMA DE TESTE}

Para fornecer dados ao software PIMS foi utilizada uma planta didática de controle de temperatura e nível de tanques, presente no laboratório de automação Industrial do Instituto Federal de Minas Gerais (IFMG) campus Formiga, conforme Figura 5. Foi utilizado como controla-

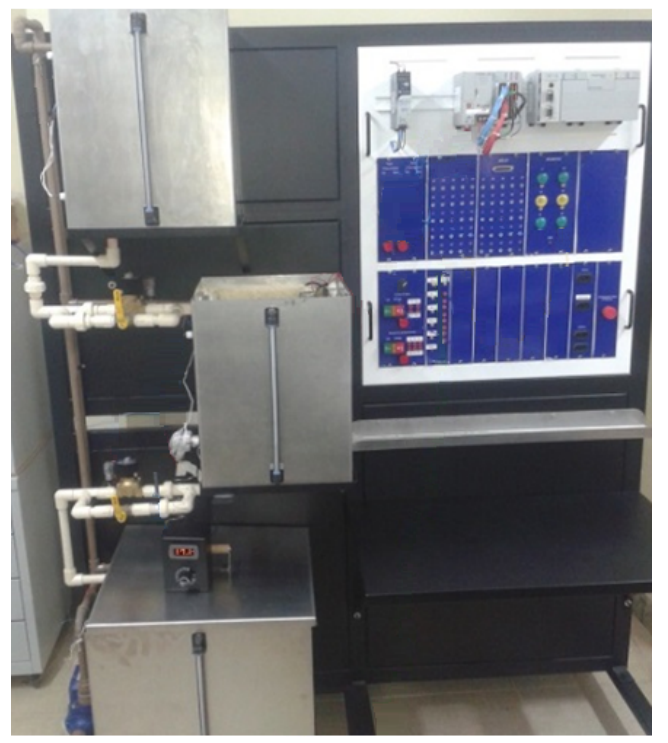

Figura 5. Planta didática de controle de temperatura e nível de tanques.

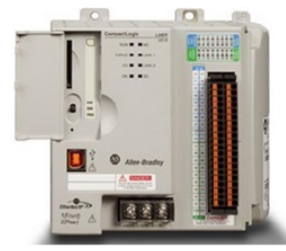

Figura 6. CLP modelo CompactLogix5370 L2 da empresa Rockwell Automation. Fonte: (Rockwell, 2019). dor do processo o CLP CompactLogix 5370 L2 (Rockwell Automation), o qual pode ser observado na Figura 6.

O programa utilizado para promover a comunicação entre o CLP e o computador é o RSLinx da empresa Rockwell Automation. Este software disponibiliza os dados do CLP em padrão OPC e, portanto, é o Servidor OPC utilizado no sistema de teste.

A configuração de uma aplicação como fonte de dados OPC consiste em criar uma conexão (Figura 7a), associando o programa do CLP com o endereço da CPU do CLP (path). A Figura 7b mostra o programa do CLP "Treinamento Básico" (à esquerda da Figura 7b) sendo associado ao CLP que está no caminho: "02, RSLogix 5000 Emulator, Treinamento Basico" (à direita da Figura 7b).

\subsection{Configuração de rede do sistema de teste}

Foi montada uma configuração de rede para testar o software PIMS desenvolvido neste trabalho. A rede é constituída de três sistemas:

- CLP (Processo industrial);

- PC Servidor (Configuração do software PIMS);

- Visualização do software PIMS.

A primeira parte da rede é o CLP. Ele realiza a aquisição dos dados do processo industrial por meio de sensores. A segunda parte é um computador (PC), utilizado para as funções de Servidor OPC, Cliente OPC, Banco de Dados e Servidor IIS (hospeda o Web Site do software PIMS). A terceira parte é composta pelos dispositivos de acesso ao Web Site, que no caso do sistema de teste é um notebook.

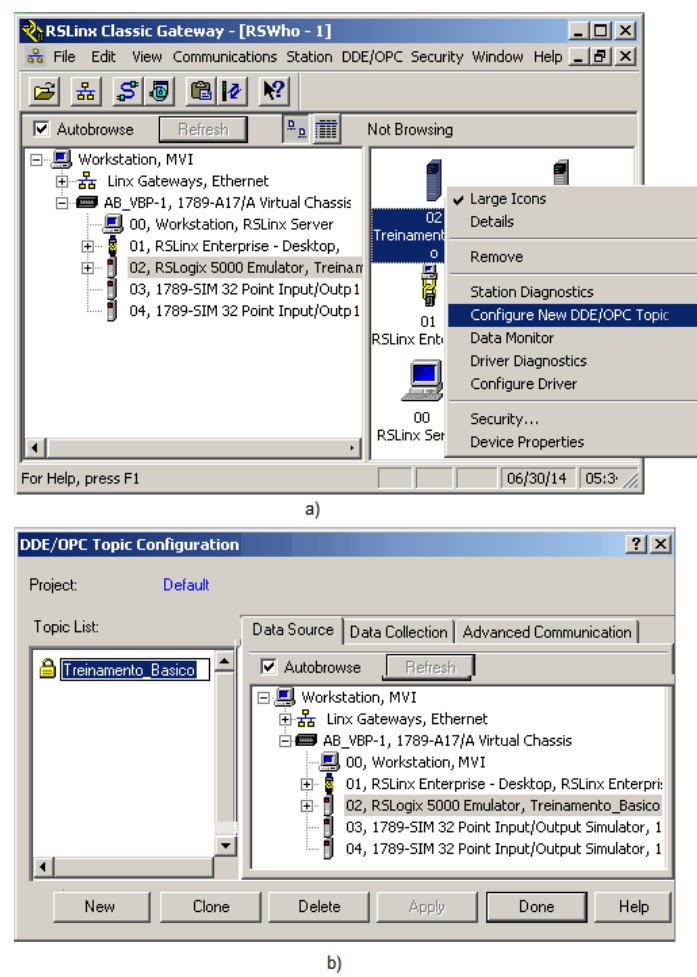

Figura 7. Configuração do Servidor OPC: a) Adicionando Conexão OPC e b) Associando o CLP ao programa Logix5000. 


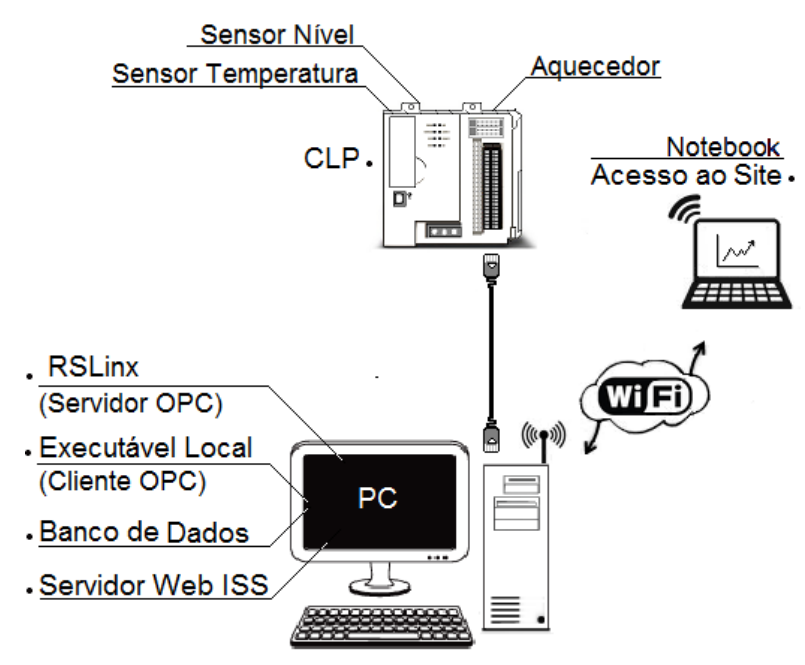

Figura 8. Sistema de teste e configuração da rede.

As três partes podem ser observadas no diagrama da Figura 8 .

A configuração de rede utilizada pode ser alterada para aplicação em um processo industrial, permitindo utilizar, por exemplo, o Servidor OPC e o Cliente OPC em um Computador e o Banco de dados e o Servidor IIS em outro computador.

\section{ANÁLISE ECONÔMICA DO SOFTWARE PIMS}

Comparar historiadores de processo não é uma tarefa fácil, pois muitos fatores devem ser levados em conta como, por exemplo, as funcionalidades básicas de um historiador na coleta, compressão, armazenamento e recuperação de dados, as ferramentas funcionais incorporadas ao historiador e também a utilização dos padrões da indústria como a comunicação OPC. A Tabela 1 (na Seção 1) mostrou os preços dos historiadores PIMS do mercado.

O software PIMS desenvolvido neste trabalho possui características compatíveis com os grandes historiadores do mercado e foi capaz de oferecer soluções como: obtenção de dados por padrão OPC; armazenamento das informações em banco de dados; sem limitação de tags; visualização de gráficos e tabelas; relatórios nos formatos PDF, XLS e DOC e visualização dos dados pelo navegador $W e b$. Essas características mostram que o software PIMS desenvolvido é tecnicamente competitivo com os seus principais concorrentes.

Além das funcionalidades um fator de extrema relevância para as empresas que adquirem esse tipo de produto é o preço a se pagar por ele. O custo de desenvolvimento do software PIMS consiste no tempo de engenharia gasto no seu desenvolvimento. Considerando o piso salarial de um Engenheiro como 8,5 vezes o salário mínimo (CONFEA, 1994) e o salário mínimo na data atual como $\mathrm{R} \$ 998,00$ (Brasil, 2018) então o salário de engenharia é igual a $\mathrm{R} \$$ $8.483,00$ por mês. Gastando o período de seis meses de desenvolvimento no software PIMS o Custo de Desenvolvimento (CD) fica conforme a Equação (1):

$$
C D=(6 \times R \$ 8.433,00)=R \$ 50.898,00
$$

Para cada software PIMS vendido será necessário instalar e configurar o sistema na empresa cliente. O tempo de instalação varia com a quantidade de variáveis a serem armazenadas. O tempo médio de instalação e configuração é de uma semana. Cada sistema terá um custo operacional de $\mathrm{R} \$ 3.000,00$. Isso inclui o valor de $\mathrm{R} \$ 2.108,25$ para o desenvolvedor, despesas com transporte para deslocamento até o cliente e alimentação durante uma semana. Os valores que um investidor desse sistema irá receber está de acordo com a Equação (2).

$$
\begin{array}{cc} 
& \text { Valor }=n \times(P S-C O)-C D \\
\text { onde: } & \\
n & - \text { Número de sistemas PIMS vendidos. } \\
P S & - \text { Preço cobrado por cada sistema. } \\
C O & - \text { Custo Operacional. }
\end{array}
$$

Com toda a solução do software PIMS sendo oferecida por $\mathrm{R} \$ 8.000,00$ o retorno do investimento virá na venda do $11^{\circ}$ software PIMS, Equação (3). Após essa venda o investidor consegue um lucro de $220 \%$ por cada sistema vendido.

$$
\text { Valor }=11 \times(8000-3000)-50898=R \$ 4.102,00
$$

O FactoryTalk Historian (versão com limitações de tags) é o historiador com menor preço dentre os listados na Tabela 1. A solução completa do software PIMS desenvolvido neste trabalho (com licença, instalação e suporte) pode ser adquirida por um preço inferior ao dos demais concorrentes. Além disso, o preço do FactoryTalk Historian se refere apenas à licença de utilização. Para configurá-lo é necessária uma empresa de automação especializada em sistemas PIMS.

\section{CONCLUSÕES}

Este trabalho mostrou que as informações do processo produtivo podem auxiliar os setores corporativos de uma indústria na tomada de decisões estratégicas. A manufatura inteligente conecta a informações dos diversos setores de uma empresa e evita ilhas de informação, auxiliando no controle e gerenciamento do processo produtivo.

A comunicação OPC é o padrão mais utilizado por fabricantes de equipamentos para automação e controle de sistemas, assim, a utilização do OPC no software PIMS desenvolvido neste trabalho o torna compatível com grandes historiadores do mercado. Os testes mostraram que o cliente OPC do software PIMS pode se conectar aos diferentes Servidores OPC, devido à padronização da comunicação OPC. Essa característica o torna interoperável em sistemas de automação de diferentes fornecedores.

Por meio do software executável local, detalhado na seção 2.1, pôde-se configurar os Servidores e Itens OPC e também cadastrar os usuários para acessar o Web Site. Ao se fazer os testes, foi possível observar uma característica de flexibilidade em relação à arquitetura de rede, onde as partes não precisam estar funcionando, necessariamente, em um só computador. Isso permite que o sistema se adapte melhor à configuração de rede da indústria que utilizar o software PIMS.

No Web Site foi possível exibir gráficos de linha, coluna e de pizza, inclusive saber a porcentagem de tempo de 
parada de uma máquina ou equipamento. Os relatórios podem ajudar os gestores de uma empresa na elaboração de documentos técnicos embasados nas informações do processo.

A análise de custos e funcionalidades, em comparação com os principais concorrentes, mostrou que o software PIMS desenvolvido neste trabalho possui as principais características dos melhores historiadores comercializados e pode ser vendido a um preço inferior, ponto importante para a proposta de oferecer esta solução às empresas de pequeno e médio porte.

Além dos ganhos quantitativos houve também ganhos qualitativos como, por exemplo, melhor organização dos dados, maior controle do processo, rapidez no acesso aos dados e também alta disponibilidade da informação de chão de fábrica.

Sugere-se, como trabalho futuro, a execução de uma avaliação de desempenho do software descrito neste trabalho. Isso pode ser realizado para efeito de comparação e completude, verificando se o sistema suporta grande volume de dados em curtos períodos de tempo. Além disso, podese pesquisar uma solução para o problema que acontece ao utilizar intervalos pequenos de coleta de dados, o que pode degradar o sistema. Uma proposta para isso é estudar a utilização de soluções distribuídas e escaláveis.

\section{AGRADECIMENTOS}

Agradecemos ao Instituto Federal de Minas Gerais (IFMG) campus Formiga por disponibilizar o laboratório de automação, a planta didática de controle de temperatura e nível de tanques e toda a estrutura necessária ao desenvolvimento desta pesquisa.

\section{REFERÊNCIAS}

Brasil (2018). Decreto de Lei assinado pelo Presidente Jair Bolsonaro em suas atribuições institui o salário mínimo do ano de 2019 como $R \$ 998,00$.

Carvalho, F.B., Torres, B.S., Fonseca, M.O., and Seixas, C.F. (2003). Sistemas PIMS - conceituação, usos e benefícios. VII Seminário de Automação de Processos da Associação Brasileira de Metalurgia e Materiais ABM, Santos, 1(4).

CONFEA (1994). O salário mínimo profissional! Uma conquista! Regulamentação do salário mínimo profissional de Engenheiros, Arquitetos e Agrônomos.

Control (2014). Global Online Comumunity of Automation professionals. OSI PI Versus Aspen Info Plus 21.

Dang, T. (2007). Integration of power plant information system with business information system in the open electricity market: challenges and solutions. In 2007 5th IEEE International Conference on Industrial Informatics, volume 2, 1209-1213.

Jazdi, N. (2016). Dynamic calculation of the reliability of factory automation applications: Industry 4.0 applications. In 2016 IEEE International Conference on Automation, Quality and Testing, Robotics (AQTR), 16.

Kao, Y.T., Chang, S.C., Blue, J., and Dauzère-Pérès, S. (2016). Generalized overall equipment effectiveness for integrated scheduling and process control. In 2016 International Symposium on Semiconductor Manufacturing (ISSM), 1-4.

Mesta (2014). Client OPC with C\#. Biblioteca de interpretação do Protocolo OPC.

Microsoft (2019). SQL Server Express Edition, software de acesso ao banco de dados. Disponivel em "www. microsoft.com", acessado em 02 de março de 2019.

OPC Foundation (2018). OPC Unified Architecture: The Interoperability Standard for Industrial Automation, 2 edition.

Rockwell, A. (2019). Controladores CompactLogix $53701769 . \quad$ Disponível em "http://ab. rockwellautomation.com/", acessado em: 02 de março de 2019.

Seixas, C.F. (2005). PIMS - Process Information Management System - Uma introdução. Universidade Federal de Minas Gerais. Notas de aula.

Shimanuki, Y. (1999). OLE for process control (OPC) for new industrial automation systems. In IEEE International Conference on Systems, Man, and Cybernetics, volume $6,1048-1050$ vol.6.

Sousa, S.M. and Santos, M.M. (2014). Desenvolvimento de um sistema PIMS com comunicação OPC e acesso por navegador web. Monografia apresentada ao Instituto Federal de Minas Gerais (IFMG) campus formiga em 18 de Agosto.

Stair, R. and Reynolds, G. (2002). Princípios de sistemas de informação: uma abordagem gerencial. LTC.

Tan, V.V., Yoo, D., and Yi, M. (2007). Modern distributed data acquisition and control systems based on opc techniques. In 14th Annual IEEE International Conference and Workshops on the Engineering of Computer-Based Systems, $115-122$.

Tierney, S. (2013). Data Logging Options For CompactLogix and ControlLogix. FactoryTalk Historian SE. 